\title{
Investigating Motivation for Physical Activity among Minority College Females Using the BREQ-2
}

\author{
Gherdai Hassel ${ }^{\mathrm{a}}$, Jeffrey John Milroy ${ }^{\mathrm{a}}$, and Muhsin Michael Orsini ${ }^{\mathrm{a}}$
}

\begin{abstract}
Adolescents who engage in regular physical activity experience increased academic performance and grades, improved academic behavior, such as time on task, as well as an increase in other factors that influence academic achievement. Despite physical activity recommendations, regular engagement in physical activity is still an issue among American adolescents. Furthermore, physically inactive lifestyles are highest among minority populations, lower socioeconomic groups, and women. Self Determination Theory provides a basis for investigating the cognitive, affective and motivational processes of physical activity. At present time, there is a dearth of research exploring motivation of physical activity among minority female college students. Therefore, the purpose of this study was to investigate the type of motivation that is most strongly related to moderate physical activity, vigorous physical activity, strength training and stretching among college-aged African American females in an effort to consider ways to improve physical activity promotion efforts on a college campus. Students enrolled in a required basic studies physical education and wellness course at a southeastern state university were invited to participate in data collection activities. Participants were invited to complete the modified Behavioral Regulation in Exercise Questionnaire (BREQ-2), a 19-item questionnaire designed to measure motivation for physical activity. Of the 1422 college students that agreed to participate, 54 identified themselves as a non-Caucasian female. Results offered limited support for the hypothesis that those who are extrinsically motivated would engage in more physical activity. Nevertheless, a required physical activity class for college students could potentially provide an opportunity for those who would otherwise be unmotivated for physical activity to engage in regular physical activities.
\end{abstract}

Keywords: Physical activity motivation; minority; females; college students

\section{Introduction}

According to the Physical Activity Guidelines for Americans released in 2008, physical activity is defined as "bodily movement that enhances health"(USDHHS, 2008). The PAG report and Healthy People 2020 (USDHHS, 2008, 2009) underscore the necessity of regular physical activity and its health-related benefits. Some benefits of regular physical activity for men and women include but are not limited to weight control, reduction of risk for cardiovascular disease, type 2 diabetes, metabolic syndrome and some cancers, bone and muscle strengthening, and improvements on mental health and mood (CDC, 2011b). Despite the recommendations by the $\mathrm{CDC}$, a lack of regular physical activity continues to be a growing issue in the United States. Negative disease outcomes are directly related to sedentary lifestyles, lack of exercise and poor diets of the growing inactive U.S population (Haskell et al., 2007).

Regular physical activity for college aged individuals also yields benefits. According to the Centers for Disease Control and Prevention (CDC), students who engage in 75150 minutes of aerobic activity each week experience increased academic performance, improved academic behavior (e.g., time on task), and improved cognitive function (e.g., concentration and attentiveness) in the classroom (CDC, 2011b). However, just as the issue is prevalent on a national scale, physical inactivity is a growing health problem among college-aged individuals as well.

A population at greater risk for physical inactivity are females, and specifically for the purpose of this study, minority female college students. Despite well publicized recommendations, African American women participate in less physical activity than any other group in college. This is not surprising, considering that in the United States physically inactive lifestyles are highest among minority populations, lower socioeconomic groups, and women (CDC, 2011a). These disparities persist even when The Healthy People 2020 indicates one of its two overarching goals is to eliminate health disparities (USDHHS, 2009). African American adults report poor or fair health more often than Caucasian and nonHispanic populations. Ethnic minority populations show patterns of behaviors related to physical activity and health that are quite different from the total U.S. population (CDC, 2011a; Ferrara, 2009). In effort to monitor the health status of minority populations the CDC created the Racial and Ethnic Approaches to Community Health (REACH U.S), an organization that sponsors the Risk Factor Survey in minority communities (CDC, 2011a). The results from the survey conducted in 2009 indicated that among the four minority communities, blacks had the highest median percentage of persons who reported engaging in no leisure-time physical activity (28.5\% in men and $31.6 \%$ in women) (CDC, 2011a). A much lower percentage of black women met physical activity recommendations in almost all communities compared with that in the corresponding county, or state (CDC, 2011a).

With such low percentages of African American women engaging in physical activity, despite the benefits of doing so, there is a need to further understand 'why' people exercise. The lack of physical activity and influence of motivation on college-aged women is especially important to consider because of its prevalence and under exploration. Of the research that has investigated motivation of physical activity, fewer studies were conducted on women and even fewer included information about minority women (Eyler et al., 2002); given this, more research and study is needed 
(DeLong, 2006; Kilpatrick et al., 2005; Wilson \& Rodgers, 2004). Therefore, the purpose of this study was to investigate the type of motivation that is most strongly related to moderate and vigorous physical activity, strength training, and stretching among college-aged African American females.

\section{Background}

Self Determination Theory (SDT) is practical when attempting to better understand the cognitive, affective and motivational processes of physical activity (Deci \& Ryan, 1985, 1991, 2000). According to Deci and Ryan, "to be motivated means to be moved by something" (1985). Physical activity motivations are influenced by many factors; SDT provides the basis for better understanding physical activity motives (DeLong, 2006; Wilson \& Rodgers, 2004). In SDT, motivation is conceived as three distinct states, (1) Amotivation, (2) a continuum of Extrinsic Motivation, and (3) Intrinsic Motivation (Deci \& Ryan, 1985).

Intrinsic motivation refers to doing something because it is inherently enjoyable (Deci \& Ryan, 1985). In exercise, intrinsic regulation involves exercising for the enjoyment and satisfaction connected with the behavior itself; it is the most autonomous form and highest level of exercise motivation (Wilson \& Rodgers, 2004). Those who are inherently motivated to exercise are not attempting achieve a result; rather they exercise because of pure enjoyment. Intrinsic regulation at its highest degree is self-determination and evidence that suggests that those who have a high degree of self-determination engage in more physical activity (Deci \& Ryan, 1985; DeLong, 2006).

Extrinsic motivation refers to doing something because it leads to a separable outcome (Deci \& Ryan, 1985). Extrinsic motivation is characterized by four types of regulation: (1) External, (2) Introjected, (3) Identified and, (4) Integrated regulation. Extrinsic motivation also involves internalization and integration of values and behavioral regulations (Deci \& Ryan, 2000). Internalization includes taking in a value or regulation, while integration is the process by which individuals more fully adopt the regulation into their own personal routine. In other words, internalization is less of an adopted activity than intrinsic identity trait (Deci \& Ryan, 2000). The continuum concept of internalization describes how one's motivation for behavior can range from amotivation or discouragement, to compliance, to active personal commitment.

According to Kilpatrick (2005), 30\% of college-aged students who engage in physical activity are active for extrinsic reasons; an even smaller proportion of these students are young women. Findings suggest that students, who are physically active to achieve some external reward, rarely enjoy the routine. Those who are mostly extrinsically motivated tend to be less self-determined than those who are intrinsically motivated (Deci \& Ryan, 1985; DeLong, 2006).

Additionally, Kilpatrick and colleagues (2005) explored the relationships between motivation and types of physical activity among college students. Results indicate that participants' motivations to engage in sport differed from motivations to engage in exercise. Kilpatrick and colleagues found that participants who were more sport motivated rated enjoyment, competition and challenge as their highest motives for physical activity. Participants who were more exercisemotivated rated appearance, strength, endurance, stress management, and weight management; all of which are extrinsic factors. These findings support the idea that sport participation is driven by intrinsic factors and exercise is motivated by extrinsic factors, further indicating that those who are intrinsically motivated are more active (Kilpatrick et al., 2005).

\section{Experimental Procedures}

\section{Recruitment \& Data Collection}

In spring of 2010, after receiving institutional review and board approval, 1422 students enrolled in a mandatory physical education and wellness course were sent an email inviting them to participate in this research study. They were asked to complete a web-based survey related to motivation and physical activity. The initial electronic invitation contained information related to the purpose of the research, informed consent for participation, and a direct link to the survey. Once the student clicked survey link, no other identifying information was collected; therefore all data sets included deidentified data which completely eliminated the possibility of connecting survey results back to participants. Participants were informed that the survey would only remain open for two weeks, and that clicking the direct link to the survey implied their consent to participate. In an attempt to increase participation two email reminders were sent over the course of the two-week data collection period.

\section{Instruments}

Subsequent to demographic questions, participants were instructed to complete items of the modified Behavioral Regulation In Exercise Questionnaire (BREQ-2). BREQ-2 is a 19-item questionnaire designed to measure five subscales, as described above, of physical activity motivation: Amotivation, External Regulation, Introjected Regulation, Identified Regulation, and Intrinsic Regulation. See Table 1 for an illustration of the type of questions asked of participants. All BREQ-2 items used a five-point response scale representing the following options: 0- Not true for me, 2- Sometimes true for me, 4-Very true for me.

Table 1: BREQ-2 Factors and Items

\begin{tabular}{cc}
\hline Factor (No. of Items) & Example Item \\
\hline Amotivation (5) & I don't see why I should have to exercise \\
\hline External Regulation (4) & I exercise because other people say I should \\
\hline Introjected Regulation (3) & I feel guilty when I don't exercise \\
\hline Identified Regulation (4) & I value the benefits of exercise \\
\hline Intrinsic regulation (4) & I exercise because it's fun \\
\hline
\end{tabular}


Data Analysis

Using SPSS 20.0 descriptive statistics were calculated to summarize demographic data elicited from participants. Afterward Pearson's bivariate correlation coefficients were generated to examine relationships and interrelationships among each of the five BREQ-2 factors and four dependent variables (days of moderate physical activity, days of vigorous physical activity, days of strength training, and days of stretching).

\section{Results}

\section{Participants}

All students enrolled in the required basic studies physical education and wellness course were invited to participate in data collection activities $(N=1422)$. Fifty-four percent $(n=762)$ of students agreed to participate and completed a confidential online survey. Of the 762 that completed the online survey, $7.1 \%$ identified themselves as a non-Caucasian female $(n=54)$ and were included in data analysis procedures. Table 2 presents demographic information about the participants. Most participants were 1819 years-old, full time students, unemployed, and either in their first or second year of college.
Table 2: Participant Demographics

\begin{tabular}{cc}
\hline Variable & Percent (\%) \\
\hline \multicolumn{1}{c}{ Age } \\
\hline $18-19$ & 63.8 \\
\hline $20-21$ & 16.4 \\
\hline $22+\quad 16.2$ \\
\hline \multicolumn{2}{c}{ Student Status } \\
\hline Full-time \\
\hline Part-time & 98.2 \\
\hline Hours employed \\
\hline $0 \quad$ \\
\hline $1-9$ & 60 \\
\hline $10-19$ & 10.9 \\
\hline $20-29$ & 16.4 \\
\hline $30-39$ & 5.5 \\
\hline 40+ & 1.8 \\
\hline Academic Standing \\
\hline First year & 5.4 \\
\hline Second year & 38.2 \\
\hline Third year & 38.2 \\
\hline Fourth year & 12.7 \\
\hline Fifth year & 9.1 \\
\hline
\end{tabular}

Table 3: Correlation Coefficients

\begin{tabular}{|c|c|c|c|c|c|c|}
\hline & & Amotivation & $\begin{array}{c}\text { External } \\
\text { Regulation }\end{array}$ & $\begin{array}{c}\text { Identified } \\
\text { Regulation }\end{array}$ & $\begin{array}{l}\text { Introjected } \\
\text { Regulation }\end{array}$ & $\begin{array}{c}\text { Intrinsic } \\
\text { Regulation }\end{array}$ \\
\hline & Sig. (2-tailed) & .000 & .000 & .000 & .000 & \\
\hline & $\mathrm{N}$ & 55 & 55 & 55 & 55 & 55 \\
\hline \multirow{3}{*}{ Moderate PA } & Pearson Correlation & $.329^{*}$ & $.283^{*}$ & $.280^{*}$ & .145 & .109 \\
\hline & Sig. (2-tailed) & .014 & .037 & .038 & .291 & .427 \\
\hline & $\mathrm{N}$ & 55 & 55 & 55 & 55 & 55 \\
\hline \multirow{3}{*}{ Vigorous PA } & Pearson Correlation & $.448^{* * *}$ & .155 & .261 & .224 & .214 \\
\hline & Sig. (2-tailed) & .001 & .259 & .054 & .101 & .117 \\
\hline & $\mathrm{N}$ & 55 & 55 & 55 & 55 & 55 \\
\hline \multirow{3}{*}{$\begin{array}{l}\text { Strength } \\
\text { Training }\end{array}$} & Pearson Correlation & $.443^{* * *}$ & .230 & $.279^{*}$ & $.295^{*}$ & .230 \\
\hline & Sig. (2-tailed) & .001 & .091 & .039 & .029 & .091 \\
\hline & $\mathrm{N}$ & 55 & 55 & 55 & 55 & 55 \\
\hline \multirow{3}{*}{ Stretching } & Pearson Correlation & $.400^{* * *}$ & .253 & $291^{*}$ & .257 & .131 \\
\hline & Sig. (2-tailed) & .002 & .062 & .031 & .058 & .340 \\
\hline & $\mathrm{N}$ & 55 & 55 & 55 & 55 & 55 \\
\hline
\end{tabular}

**Correlation is significant at the 0.01 level

*Correlation is significant at the 0.05 level

Table 3 presents correlation coefficients that describe the direction and magnitude of relationships among 5 independent variables (BREQ-2) and 4 dependent variables (behavior). Amotivation is significantly correlated with all 4 behavioral dependent variables $(p<.05)$. External Regulation is significantly correlated with 1 behavioral dependent variable - Moderate Physical activity $(p<.05)$. Identified regulation is significantly correlated with 3 behavioral independent variables - Moderate Physical Activity, Strength Training and Stretching $(p<.05)$. Introjected Regulation is significantly correlated with 1 behavioral dependent variable - Strength Training $(p<.05)$. Intrinsic Regulation is not significantly correlated with any of the behavioral dependent variables. 


\section{Discussion}

Findings suggest relationships among 5 independent variables (BREQ-2) and 4 dependent variables (behavior). Results offered limited support for the hypothesis that those who are extrinsically motivated would engage in more physical activity. Results from the study indicate that amotivation was moderately associated to vigorous physical activity. This deviates from Deci and Ryan's Selfdetermination theory and motivation. Whereas, SDT suggests that those who were intrinsically motivated would exercise vigorously, the data shows otherwise. This result could be attributed to the fact that data was collected from a group of participants who were enrolled in a mandatory physical education course; therefore, they simply complied with course requirements.

Aforementioned, extrinsically motivated behaviors are characterized by 4 types of regulation: external regulation, introjected regulation, identified regulation and integrated regulation (Deci \& Ryan, 2000). SDT theory states that, those who are intrinsically motivated to do something for the love of the activity; whereas those who are extrinsically motivated do something for a separable outcome. However, the results of this study indicate that amotivation is significantly correlated with all 4 behavioral dependent variables, Results show that those who were amotivated engaged in physical activity the most. External Regulation is significantly correlated with 1 behavioral dependent variable - Moderate Physical activity. Identified regulation is significantly correlated with 3 behavioral independent variables Moderate Physical Activity, Strength Training and Stretching. Intrinsic Regulation is not significantly correlated with any of the behavioral dependent variables. In fact, contrary to SDT, results of this study suggest that, under appropriate conditions, both amotivation and extrinsic motivation are related to various types of physical activity.

\section{Limitations}

Limitations related to self-report data apply to this study; however, strategies were used to reduce the likelihood of eliciting socially desirable responses. Survey items did not elicit sensitive information and participants were informed that their responses would be kept confidential.

Another potential limitation was the small sample size, suggesting that the sample may not accurately represent the larger population. Although this may be true, from conception this study was intended to be an initial and preliminary investigation into physical activity motivation held by nonCaucasian college females, and therefore was warranted.

In this study, an unintended limitation involves recruitment. Students were invited to participate in data collection procedures two weeks after the start of the semester, by which time they had already begun participating in required physical activity labs. Ultimately this eliminated most sedentary students; i.e., most everyone was complying with course requirements and engaging in various forms of physical activity. Recruitment procedures may have limited the findings of this research study; however, findings suggest that even amotivated students can by "encouraged" to be physically active, which although unintended was in important finding.

Finally, intercorrelations among the 5 independent variables (BREQ-2) suggest that SDT may not be the best theoretical perspective for understanding motivational predictors among this population. Additional research ought to investigate the reliability and validity of BREQ-2 for assessing motivation among minority females in college.

\section{Implications for future research}

In conclusion, prior research indicates that college students, especially women and minority women in college, are not meeting the recommended guidelines for physical activity established by the CDC. While mandatory physical activity programs have increased the amount of physical activity students engage in, they have not been effective in fostering students to adopt physical activity behaviors on their own. More research is needed to determine effective intervention efforts that can be long lasting. A plethora of research opportunities exist for researchers, as minority women represent the highest group to report sedentary lifestyle in the U.S (CDC, 2011a) and have rarely been included in research and intervention efforts for physical activity. Based on these limitations, studies like this should be replicated in which participants can be surveyed with the BREQ instrument, followed for longer periods of time, and could potentially be given to upperclassmen as well to provide more insight to the motivations for physical activity engagement. If determinants that influence physical activity engagement for this group can be clearly identified, then perhaps more effective intervention efforts can be developed and implemented.

\section{Implications for future practice}

The dependent variables examined in this study did not offer clear identification of appropriate intervention strategies. However, colleges and university settings are an ideal setting for promoting physical activity, exercise and weight management (Ferrara, 2009). Required physical activity classes for first year college students could potentially provide students with the motivation they need to adopt physical activity behaviors on their own as they progress through college. In the college and university setting lies hope to convert sedentary students to moderate and vigorous physical activity engagers. Evidence from prevention science and research should direct practice, especially as it relates to physical activity promotion and intervention efforts. Findings of this research study suggest that non-Caucasian college females may need to be told to be physically active. Therefore, required physical activity courses may be most beneficial for people who are typically amotivated for physical activity, and could potentially encourage them to continue to be physically active for a lifetime.

\section{References}

CDC. (2011a). Minority Health Surveillance - REACH U.S. 2009. Retrieved from http://www.cdc.gov/features/dsreachus/

CDC. (2011b). Physical Activity and Health. Retrieved from http://www.cdc.gov/physicalactivity/everyone/health/

Deci, E., \& Ryan, R. (1985). Intrinsic motivation and selfdetermination in human behavior: New York: Springer.

Deci, E. L., \& Ryan, R. M. (1991). A motivational approach to self: Integration in personality. Paper presented at the Nebraska symposium on motivation. 
Deci, E. L., \& Ryan, R. M. (2000). The" what" and" why" of goal pursuits: Human needs and the self-determination of behavior. Psychological inquiry, 11(4), 227-268.

DeLong, L. L. (2006). College Students' Motivation for Physical Activity (Doctoral dissertation) University of Louisiana.

Eyler, A. A., Vest, J. R., Sanderson, B., Wilbur, J., MatsonKoffman, D., Evenson, K. R., Young, D. R. (2002). Environmental, policy, and cultural factors related to physical activity in a diverse sample of women: the Women's Cardiovascular Health Network Projectintroduction and methodology. Women \& Health, 36(2), 115.

Ferrara, C. (2009). The college experience: Physical activity, nutrition, and implications for intervention and future research. Journal of Exercise Physiology, 12(1), 23-35.

Haskell, W. L., Lee, I., Pate, R. R., Powell, K. E., Blair, S. N., Franklin, B. A., Bauman, A. (2007). Physical Activity and public Health: Updated Recommendation for Adults From The American College of Sports Medicine and The American Heart Association. Medicine and science in sports and exercise, 39(8), 1423.

Kilpatrick, M., Hebert, E., \& Bartholomew, J. (2005). College Students' Motivation for Physical Activity: Differentiating Men's and Women's Motives for Sport Participation and Exercise. Journal of American College Health, 54(2), 8794.

USDHHS. (2008). Physical Activity Guidelines for Americans: Be Active, Healthy and Happy! (ODPHP Publication No. U0036).

USDHHS. (2009). Healthy People 2020: The Road Ahead. Journal of Environemental Health, 70(10).

Wilson, P. M., \& Rodgers, W. M. (2004). The Relationship Between Perceived Autonomy Support, Exercise Regulations and Behavioral Intentions in Women. Psychology of Sport and Exercise, 5(3), 229-242. 